\title{
GENERALIZATIONS OF HÖLDER'S INEQUALITY
}

\author{
WING-SUM CHEUNG
}

(Received 7 August 2000)

\begin{abstract}
Some generalized Hölder's inequalities for positive as well as negative exponents are obtained.
\end{abstract}

2000 Mathematics Subject Classification. 26D07, $26 D 15$.

1. Introduction. While the renowned inequality of Hölder [1] is well celebrated for its beauty and its wide range of important applications to real and complex analysis, functional analysis, as well as many disciplines in applied mathematics, it is a little less well known that it has a counterpart for negative exponents. Furthermore, it appears that nowhere in the literature contains a generalization of them to the case involving more than 2 dependent functions. It is the purpose of the present note to explore such generalizations. Interested readers are also referred to [2] for an "inverse Hölder's inequality" (for positive exponents) and its generalizations.

For the sake of convenience, we first restate the classical Hölder's inequalities here.

HÖLDERS INEQUALITY FOR POSITIVE EXPONENTS [1, page 190]. Let $p, q>1$ be real numbers satisfying $1 / p+1 / q=1$. Let $f \in \mathcal{L}^{p}, g \in \mathcal{L}^{q}$. Then $f g \in \mathcal{L}^{1}$ and

$$
\int|f g| d \mu \leq\|f\|_{p}\|g\|_{q} .
$$

HöLDER'S INEQUALITY FOR NEGATIVE EXPONENTS [1, page 191]. Let $0<p<1$ and $q \in \mathbb{R}$ be such that $1 / p+1 / q=1$ (hence $q<0$ ). If $f, g$ are measurable functions, then

$$
\int|f g| d \mu \geq\left(\int|f|^{p} d \mu\right)^{1 / p}\left(\int|g|^{q} d \mu\right)^{1 / q}
$$

unless $\int|g|^{q}=0$, in which case the right-hand side of (1.2) does not make sense.

2. The inequalities. Let $m \geq 2$ be any integer. Hölder's inequalities (1.1) and (1.2) can be generalized to the case involving $m$ functions as follows.

THeOrem 2.1 (generalized Hölder's inequality for positive exponents). Let $p_{1}, \ldots$, $p_{m}>0$ be real numbers such that $\sum_{\alpha=1}^{m} 1 / p_{\alpha}=1$. Let $f_{\alpha} \in \mathcal{L}^{p_{\alpha}}, \alpha=1, \ldots, m$. Then $\prod_{\alpha=1}^{m} f_{\alpha} \in \mathcal{L}^{1}$ and

$$
\int \prod_{\alpha=1}^{m}\left|f_{\alpha}\right| d \mu \leq \prod_{\alpha=1}^{m}\left\|f_{\alpha}\right\|_{p_{\alpha}} .
$$

Proof. We use induction on $m$. When $m=2$, we are given $p_{1}, p_{2}>0$ with $1 / p_{1}+$ $1 / p_{2}=1$. In particular, we have $p_{1}, p_{2}>1$ and so (2.1) is reduced to the classical 
Hölder's inequality (1.1). Now suppose (2.1) holds for some integer $m \geq 2$. We claim that it also holds for $m+1$. So let $p_{1}, \ldots, p_{m+1}>0$ be real numbers with $\sum_{\alpha=1}^{m+1} 1 / p_{\alpha}=1$ and let $f_{\alpha} \in \mathcal{L}^{p_{\alpha}}, \alpha=1, \ldots, m+1$. Note that, as above, we must have $p_{\alpha}>1$ for $\alpha=1, \ldots, m+1$. In particular, we have

$$
p_{1}>0, \quad \frac{p_{1}}{p_{1}-1}>0, \quad \frac{1}{p_{1}}+\frac{1}{p_{1} /\left(p_{1}-1\right)}=1
$$

Thus by the classical Hölder's inequality (1.1),

$$
\begin{aligned}
\int \prod_{\alpha=1}^{m+1}\left|f_{\alpha}\right| d \mu & =\int\left|f_{1}\right| \cdot \prod_{\alpha=2}^{m+1}\left|f_{\alpha}\right| d \mu \\
& \leq\left\|f_{1}\right\|_{p_{1}}\left[\int\left(\prod_{\alpha=2}^{m+1}\left|f_{\alpha}\right|\right)^{p_{1} /\left(p_{1}-1\right)} d \mu\right]^{\left(p_{1}-1\right) / p_{1}} \\
& =\left\|f_{1}\right\|_{p_{1}}\left[\int \prod_{\alpha=2}^{m+1}\left|f_{\alpha}\right|^{p_{1} /\left(p_{1}-1\right)} d \mu\right]^{\left(p_{1}-1\right) / p_{1}}
\end{aligned}
$$

Next, since

$$
\begin{gathered}
\frac{p_{\alpha}\left(p_{1}-1\right)}{p_{1}}>0 \text { for } \alpha=2, \ldots, m+1, \\
\sum_{\alpha=2}^{m+1} \frac{1}{p_{\alpha}\left(p_{1}-1\right) / p_{1}}=\frac{p_{1}}{p_{1}-1} \sum_{\alpha=2}^{m+1} \frac{1}{p_{\alpha}}=\frac{p_{1}}{p_{1}-1}\left(1-\frac{1}{p_{1}}\right)=1,
\end{gathered}
$$

by induction hypothesis and (2.3), we arrive at

$$
\begin{aligned}
\int \prod_{\alpha=1}^{m+1}\left|f_{\alpha}\right| d \mu & \leq\left\|f_{1}\right\|_{p_{1}}\left[\prod_{\alpha=2}^{m+1}\left(\int\left|f_{\alpha}\right|^{p_{1} /\left(p_{1}-1\right) \cdot p_{\alpha}\left(p_{1}-1\right) / p_{1}} d \mu\right)^{p_{1} / p_{\alpha}\left(p_{1}-1\right)}\right]^{\left(p_{1}-1\right) / p_{1}} \\
& =\left\|f_{1}\right\|_{p_{1}} \cdot \prod_{\alpha=2}^{m+1}\left(\int\left|f_{\alpha}\right|^{p_{\alpha}} d \mu\right)^{1 / p_{\alpha}}
\end{aligned}
$$

and so the assertion follows.

While in Theorem 2.1 it seems quite obvious on how to generalize the classical Hölder's inequality (1.1) to the situation involving $m(\geq 2)$ functions, the generalization of inequality (1.2) to the same situation is another story. There is no obvious way to achieve that. In fact, one can easily observe that there are at least two possible directions to tackle the problem. First, to try to generalize to the situation where all but one of the $p_{\alpha}$ 's are real numbers lying in the interval $(0,1)$, hence the other 
one is forced to be negative in order that $\sum 1 / p_{\alpha}=1$; and second, to the situation where all but one of the $p_{\alpha}$ 's are negative, and the other one being positive to assure $\sum 1 / p_{\alpha}=1$. Experience tells us that only one of these directions can be right. Indeed, as can be seen in the following theorem, the latter direction is the correct one. Interested readers are advised to try and work on the first direction and experience the obstruction there.

THEOREM 2.2 (generalized Hölder's inequality for negative exponents). Let $p_{1}, \ldots$, $p_{m-1}<0$ and $p_{m} \in \mathbb{R}$ be such that $\sum_{\alpha=1}^{m} 1 / p_{\alpha}=1$ (hence $0<p_{m}<1$ ). Let $f_{\alpha}$ be measurable functions for $\alpha=1, \ldots, m$. Then

$$
\int \prod_{\alpha=1}^{m}\left|f_{\alpha}\right| d \mu \geq \prod_{\alpha=1}^{m}\left(\int\left|f_{\alpha}\right|^{p_{\alpha}} d \mu\right)^{1 / p_{\alpha}}
$$

unless $\int\left|f_{\alpha}\right|^{p_{\alpha}} d \mu=0$ for some $\alpha=1, \ldots, m-1$, in which case the right-hand side of (2.7) does not make sense.

Proof. Similar to the proof of Theorem 2.1, we use induction on $m$. Clearly when $m=2$, equation (2.7) reduces to the classical Hölder's inequality (1.2). Now suppose that (2.7) holds for some integer $m \geq 2$. We claim that it also holds for $m+1$. So let $p_{1}, \ldots, p_{m}<0$ and $p_{m+1} \in \mathbb{R}$ be such that $\sum_{\alpha=1}^{m+1} 1 / p_{\alpha}=1$ and let $f_{\alpha}, \alpha=1, \ldots, m+1$, be measurable functions. Note that $0<p_{m+1}<1$. Since

$$
p_{1}<0, \quad 0<\frac{p_{1}}{p_{1}-1}<1, \quad \frac{1}{p_{1}}+\frac{1}{p_{1} /\left(p_{1}-1\right)}=1,
$$

by the classical Hölder's inequality (1.2), we have

$$
\begin{aligned}
\int \prod_{\alpha=1}^{m+1}\left|f_{\alpha}\right| d \mu & =\int\left|f_{1}\right| \cdot \prod_{\alpha=2}^{m+1}\left|f_{\alpha}\right| d \mu \\
& \geq\left(\int\left|f_{1}\right|^{p_{1}} d \mu\right)^{1 / p_{1}}\left[\int\left(\prod_{\alpha=2}^{m+1}\left|f_{\alpha}\right|\right)^{p_{1} /\left(p_{1}-1\right)} d \mu\right]^{\left(p_{1}-1\right) / p_{1}} \\
& =\left(\int\left|f_{1}\right|^{p_{1}} d \mu\right)^{1 / p_{1}}\left[\int \prod_{\alpha=2}^{m+1}\left|f_{\alpha}\right|^{p_{1} /\left(p_{1}-1\right)} d \mu\right]^{\left(p_{1}-1\right) / p_{1}}
\end{aligned}
$$

unless $\int\left|f_{1}\right|^{p_{1}} d \mu=0$. Now since

$$
\frac{p_{\alpha}\left(p_{1}-1\right)}{p_{1}}<0 \quad \text { for } \alpha=2, \ldots, m, \quad \frac{p_{m+1}\left(p_{1}-1\right)}{p_{1}}>0,
$$

and as in (2.5),

$$
\sum_{\alpha=2}^{m+1} \frac{1}{p_{\alpha}\left(p_{1}-1\right) / p_{1}}=1
$$


by induction hypothesis and (2.9), we obtain

$$
\begin{aligned}
\int \prod_{\alpha=1}^{m+1}\left|f_{\alpha}\right| d \mu & =\left(\int\left|f_{1}\right|^{p_{1}} d \mu\right)^{1 / p_{1}}\left[\prod_{\alpha=2}^{m+1}\left(\int\left|f_{\alpha}\right|^{p_{1} /\left(p_{1}-1\right) \cdot p_{\alpha}\left(p_{1}-1\right) / p_{1}} d \mu\right)^{p_{1} / p_{\alpha}\left(p_{1}-1\right)}\right]^{\left(p_{1}-1\right) / p_{1}} \\
& =\left(\int\left|f_{1}\right|^{p_{1}} d \mu\right)^{1 / p_{1}} \prod_{\alpha=2}^{m+1}\left(\int\left|f_{\alpha}\right|^{p_{\alpha}} d \mu\right)^{1 / p_{\alpha}} \\
& =\prod_{\alpha=1}^{m+1}\left(\int\left|f_{\alpha}\right|^{p_{\alpha}} d \mu\right)^{1 / p_{\alpha}}
\end{aligned}
$$

unless $\int\left|f_{\alpha}\right|^{p_{\alpha}} d \mu=0$ for some $\alpha=1, \ldots, m$.

ACKNOWLEDGEMENT. This work was supported in part by a HKU CRCG grant.

\section{REFERENCES}

[1] E. Hewitt and K. Stromberg, Real and Abstract Analysis. A Modern Treatment of the Theory of Functions of a Real Variable, second printing corrected, Springer-Verlag, Berlin, 1969. MR 43\#428. Zbl 225.26001.

[2] D. S. Mitrinović, Analytic Inequalities, in cooperation with P. M. Vasic. Die Grundlehren der mathematischen WisenschaftenIn cooperation with P. M. Vasic. Die Grundlehren der mathematischen Wisenschaften, Band 1965, Springer-Verlag, Berlin, 1970. MR 43\#448. Zbl 199.38101.

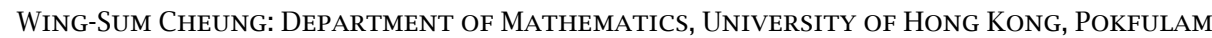
ROAD, HONG KONG

E-mail address: wscheung@nkucc. hku. hk 


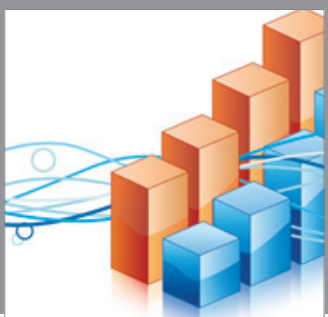

Advances in

Operations Research

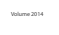

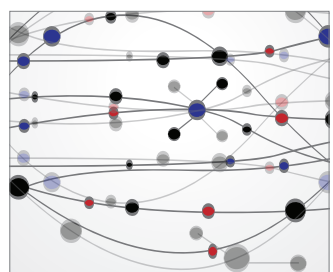

\section{The Scientific} World Journal
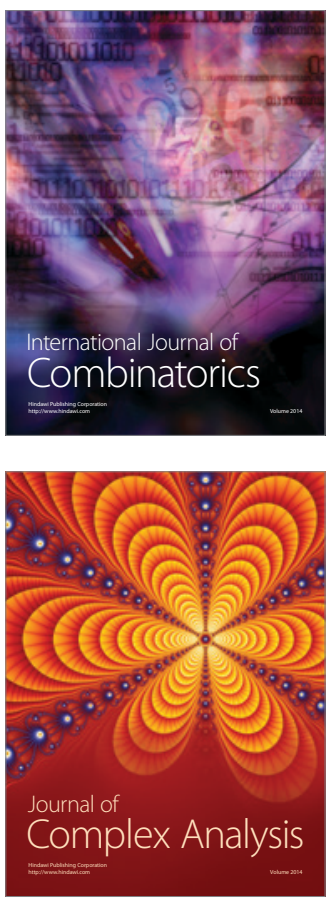

International Journal of

Mathematics and

Mathematical

Sciences
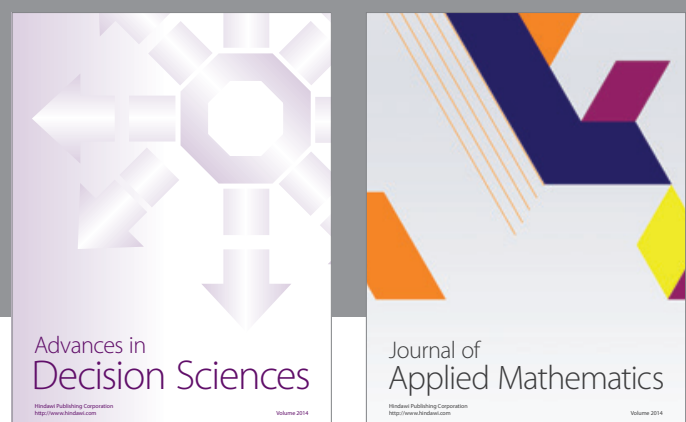

Journal of

Applied Mathematics
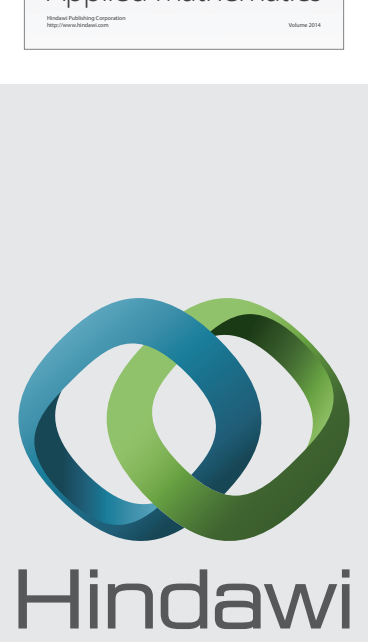

Submit your manuscripts at http://www.hindawi.com
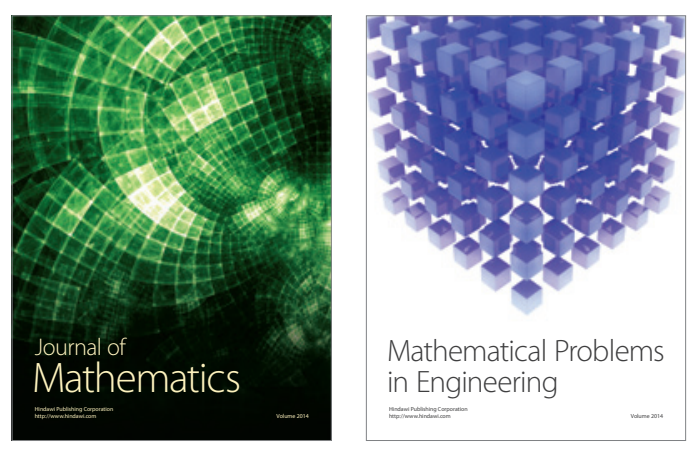

Mathematical Problems in Engineering
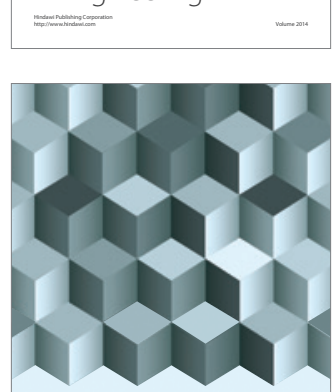

Journal of

Function Spaces
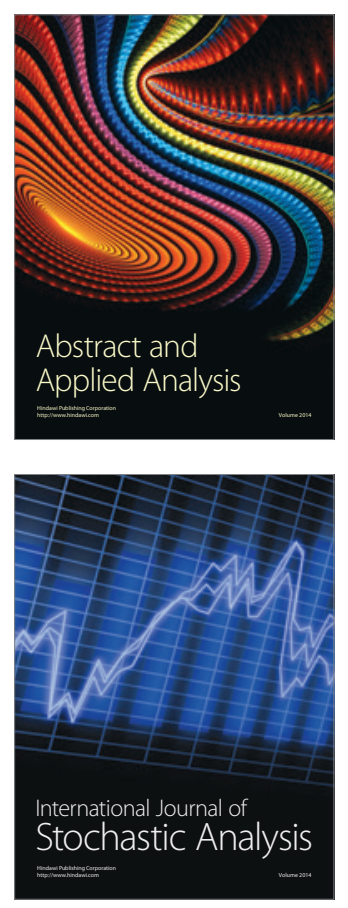

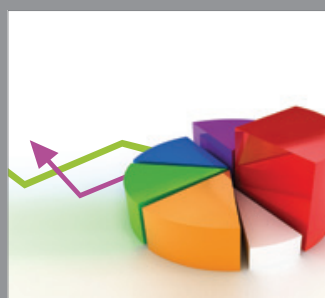

ournal of

Probability and Statistics

Promensencen
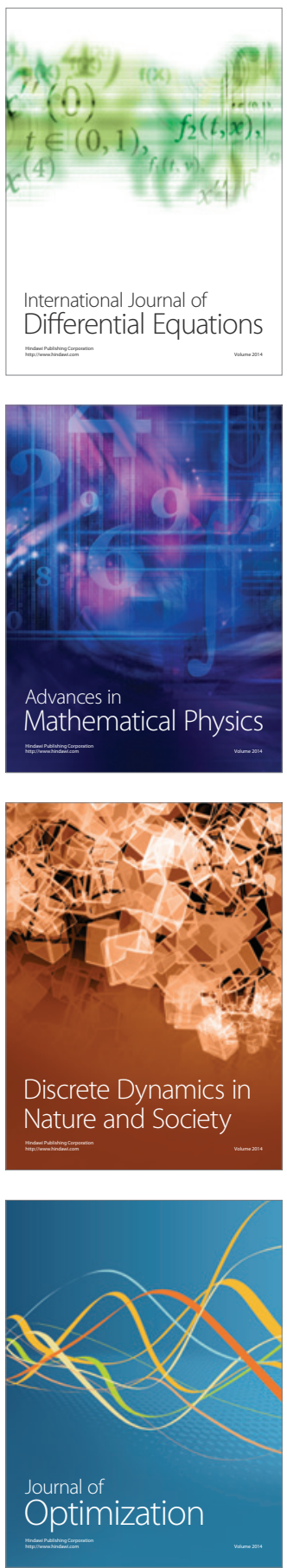